\title{
Inducible podocyte-specific deletion of CTCF drives progressive kidney disease and bone abnormalities
}

\author{
Marta Christov, ${ }^{1,2}$ Abbe R. Clark, ${ }^{3,4}$ Braden Corbin, ${ }^{1}$ Samy Hakroush, ${ }^{5}$ Eugene P. Rhee, ${ }^{1,6}$ \\ Hiroaki Saito, ${ }^{7}$ Dan Brooks, ${ }^{1}$ Eric Hesse, ${ }^{7}$ Mary Bouxsein ${ }^{1,8}$ Niels Galjart, ${ }^{9}$ Ji Yong Jung, ${ }^{3}$ \\ Peter Mundel, ${ }^{10}$ Harald Jüppner, ${ }^{1,11}$ Astrid Weins, ${ }^{3,12}$ and Anna Greka ${ }^{3,4}$ \\ 'Endocrine Unit, Massachusetts General Hospital and Harvard Medical School, Boston, Massachusetts, USA. ${ }^{2}$ Department \\ of Medicine, New York Medical College, Valhalla, New York, USA. 'Department of Medicine, Brigham and Women's \\ Hospital and Harvard Medical School, Boston, Massachusetts, USA. ${ }^{4}$ The Broad Institute of MIT and Harvard, Cambridge, \\ Massachusetts, USA. ${ }^{5}$ Department of Pathology, University of Göttingen, Göttingen, Germany. ${ }^{6}$ Nephrology Division, \\ Massachusetts General Hospital and Harvard Medical School, Boston, Massachusetts. USA. ${ }^{7}$ Heisenberg-Group for \\ Molecular Skeletal Biology, Department of Trauma, Hand \& Reconstructive Surgery, University Medical Center Hamburg- \\ Eppendorf, Hamburg, Germany. ${ }^{8}$ Center for Advanced Orthopaedic Studies, Beth Israel Deaconess Medical Center, Boston, \\ Massachusetts, USA. ${ }^{9}$ Department of Cell Biology, Erasmus MC, Rotterdam, Netherlands. ${ }^{10}$ Department of Medicine, \\ and "Pediatric Nephrology, Massachusetts General Hospital and Harvard Medical School, Boston, Massachusetts, USA. \\ ${ }^{12}$ Department of Pathology, Brigham and Women's Hospital and Harvard Medical School, Boston, Massachusetts, USA.
}

\begin{abstract}
Progressive chronic kidney diseases (CKDs) are on the rise worldwide. However, the sequence of events resulting in CKD progression remain poorly understood. Animal models of CKD exploring these issues are confounded by systemic toxicities or surgical interventions to acutely induce kidney injury. Here we report the generation of a CKD mouse model through the inducible podocytespecific ablation of an essential endogenous molecule, the chromatin structure regulator CCCTCbinding factor (CTCF), which leads to rapid podocyte loss (iCTCF ${ }^{\text {pod }-/-}$ ). As a consequence, iCTCF ${ }^{\text {pod }-/-}$ mice develop severe progressive albuminuria, hyperlipidemia, hypoalbuminemia, and impairment of renal function, and die within 8-10 weeks. CKD progression in iCTCF ${ }^{\text {pod-/- }}$ mice leads to high serum phosphate and elevations in fibroblast growth factor 23 (FGF23) and parathyroid hormone that rapidly cause bone mineralization defects, increased bone resorption, and bone loss. Dissection of the timeline leading to glomerular pathology in this CKD model led to the surprising observation that podocyte ablation and the resulting glomerular filter destruction is sufficient to drive progressive CKD and osteodystrophy in the absence of interstitial fibrosis. This work introduces an animal model with significant advantages for the study of CKD progression, and it highlights the need for podocyte-protective strategies for future kidney therapeutics.
\end{abstract}

Authorship note: $M C$ and $A R C$ contributed equally to this work.

Conflict of interest: PM is an employee of Goldfinch Biopharma. AG declares consultation services for Bristol Myers Squibb, Third Rock Ventures, and Goldfinch Biopharma.

Submitted: May 10, 2017

Accepted: January 23, 2018

Published: February 22, 2018

Reference information:

JCI Insight. 2018;3(4):e95091. https:// doi.org/10.1172/jci.insight.95091.

\section{Introduction}

Progressive chronic kidney diseases (CKDs) affect more than 500 million people worldwide and are the causes of death at an alarming rate (1). With an average survival of only 3-5 years for patients with endstage kidney disease (ESKD) treated by dialysis (worse than most cancers), and much less if not treated, progressive kidney disease is recognized as a worldwide public health problem $(1,2)$. However, we currently lack in-depth understanding of the timing of onset and progression of kidney disease. Furthermore, there are currently no reliable biomarkers for early detection of impaired kidney function, which can enable therapeutic interventions to prevent or slow disease progression.

Two universally accepted hallmarks of kidney disease progression are a decreased glomerular filtration rate (GFR), as manifested by an elevated serum creatinine level, and progressive albuminuria, the abnormal spilling of albumin into the urine as a consequence of a damaged glomerular filtration barrier $(2,3)$. Impaired renal function contributes to an abnormal regulation of mineral ion homeostasis, noticed even during the early stages of kidney disease by an increase in the circulating levels of FGF23, a phosphate-regulating hormone that reduces 1,25-dihydroxyvitamin $\mathrm{D}(1,25 \mathrm{D})$ concentrations through different 
mechanisms, thus limiting intestinal calcium absorption and accelerating the development of secondary hyperparathyroidism (HPT) (4). Parathyroid hormone (PTH) elevation triggers osteoclast-mediated bone resorption, which helps maintain normal blood ionized calcium levels for extended periods of time. However, bone resorption increases not only the release of calcium, but also of phosphate, which along with phosphate from dietary sources increases the overall phosphate burden, thereby contributing to progressively elevated serum phosphate levels.

The PTH-dependent changes in bone metabolism, referred to as CKD-mineral and bone disorder (CKD-MBD), contribute to an increased frequency of fractures in adult and pediatric CKD patients, and are associated with significant additional mortality $(5,6)$. While PTH elevation occurs relatively late during the course of CKD, increases in FGF23 occur much earlier (4). Therefore, FGF23 is increasingly recognized as an important early biomarker of impaired kidney function, which furthermore predicts kidney disease progression and is associated with cardiovascular complications and increased mortality (4).

Many studies have argued that GFR decline and CKD progression require in all cases the presence of significant tubulointerstitial fibrosis, the consequence of increased deposition of fibrillar matrix (primarily collagen) in the space between kidney tubules and peritubular capillaries due to increased activity of kidney myofibroblasts (7). However, whether kidney diseases characterized by albuminuria (which occurs in the majority of progressive kidney diseases) require interstitial fibrosis for progression to CKD and ESKD remains controversial (8-10). Moreover, there is no clear understanding as to whether elevations of PTH (and possibly FGF23) that are responsible for development of CKD-MBD occur as a consequence of overall impaired renal function or whether interstitial fibrosis resulting in tubular dysfunction is required before circulating levels of these hormones increase. Nonetheless, FGF23 and PTH levels can serve as biomarkers that herald the onset and progression of kidney disease, and could lead to early therapeutic interventions halting or slowing disease progression.

The difficulty in answering these questions and bringing greater clarity to the onset and timing of distinct progressive kidney diseases largely lies in the lack of appropriate animal models. Surgical models, such as the unilateral ureteral obstruction model to induce fibrosis (11) or the 5/6 nephrectomy model (12) lead to significant interindividual variation of phenotype and do not recapitulate many of the hallmarks of human CKD-MBD unless further stressed, for example through phosphate loading (12). In other models, the systemic toxicities of agents used to generate animal models of CKD, such as the injection of puromycin aminonucleoside to induce nephrosis, cause widespread cellular damage that is not restricted to the kidney, making it difficult to carefully dissect the sequence of pathologic events during disease progression (13). The adenine nephropathy model leads to tubulointerstitial damage (14) and thus does not reflect the most common reason for human disease, which is glomerular pathology (9).

The zinc finger DNA binding protein CCCTC-binding factor (CTCF) is considered an essential regulator of chromosomal 3D architecture $(15,16)$ and its deletion leads to cell death (17). We therefore reasoned that targeted ablation of this critical intracellular molecule in a doxycycline-inducible manner in podocytes would lead to their selective elimination. While the overexpression and activation of human diphtheria toxin in podocytes can also lead to podocyte death (18), here we chose to delete an essential endogenous molecule to avoid the possibility that overexpression of an exogenous toxin may cause unanticipated offtarget effects in podocytes or other neighboring cells.

\section{Results}

Podocyte-specific Ctcf deletion (iCTCF $F^{\text {od-l--) }}$ in adult mice causes nephrotic syndrome, kidney failure, and premature death. Heterozygous, floxed CTCF mice $\left(C t c f^{\mathrm{ft} / \mathrm{f}}\right)$ were mated with doxycycline-inducible, podocytespecific CRE-transgenic mice (iCre $\left.{ }^{\mathrm{pod}}\right)$ to generate $\mathrm{i} C r e^{\mathrm{pod}}-\mathrm{Ctc} f^{\mathrm{fut} / \mathrm{fl}}$ mice, which were then inbred to generate i $C r e^{\text {pod }}-C t c f^{1 / 1 / 1}$ and i $C r e^{\text {pod }}-C t c f^{\text {vit/wt }}$ mice (Supplemental Figure 1; supplemental material available online with this article; https://doi.org/10.1172/jci.insight.95091DS1). CTCF ablation in iCre ${ }^{\text {pod_Ctcfl/fl }}$ mice was achieved by doxycycline to induce Cre expression in podocytes starting at 6 weeks of age to generate iCTCF ${ }^{\text {pod-l- }}$ mice; doxycycline-treated $\mathrm{i} C r e^{\mathrm{pod}}-\mathrm{Ctcf}^{\mathrm{fut} / \mathrm{wt}}$ mice (wild-type; WT) mice served as controls. To evaluate the efficiency and specificity of Ctcf deletion, glomerular sections of iCTCF ${ }^{\text {pod-l- }}$ and WT mice were costained for CTCF and synaptopodin, a podocyte-specific marker (Figure 1A and Supplemental Figure 2A, arrows). One week after Cre induction, CTCF was undetectable by immunohistochemistry in podocytes of iCTCF ${ }^{\text {pod-/- }}$ mice as compared with doxycycline-treated WT controls. 
Urinary 24-hour albumin excretion, measured in metabolic cages, increased progressively in iCTCF ${ }^{\text {pod-/- }}$ mice compared with controls starting at 4 weeks after Cre induction, reaching up to $40 \mathrm{mg} /$ day at 8 weeks (Figure 1B). Consistent with the development of nephrotic syndrome, serum albumin levels decreased by 4 weeks (Figure 1C). To further characterize the hyperlipidemic state associated with the nephrotic syndrome, we performed liquid chromatography, mass spectrometry-based lipidomic analyses of nephrotic iCTCF ${ }^{\text {pod-/- }}$ mouse sera versus WT controls. At 6 weeks after Cre induction, we observed significant and progressive elevations in a wide array of lipid metabolites in the setting of nephrosis, including elevations in cholesterol esters, sphingomyelins, and phosphatidylcholines (Supplemental Figure 2, B-D and Supplemental Table 1), offering, to the best of our knowledge, a uniquely detailed lipid profile of nephrotic syndrome.

Next, we measured serum and urine markers of kidney function in control and iCTCF ${ }^{\text {pod-/- }}$ mice after 2, 4, 6, and 8 weeks of doxycycline-mediated Cre induction. As shown in Figure 1D, creatinine levels increased significantly at 8 weeks after Cre induction, while blood urea nitrogen (BUN) levels significantly increased as early as 6 weeks after Cre induction (Figure 1E). With progressive nephrosis and kidney failure, we also detected significant and progressive podocyte loss starting at 2 weeks after Cre induction (Figure 1F and Supplemental Figure 2E). In survival analyses carried out to 150 days after Cre induction, median survival was approximately 8 weeks for iCTCF ${ }^{\mathrm{pod}-/-}$ mice, and most mice had died by day 150 of life, in contrast to WT controls (Figure $1 \mathrm{G})$. In light of this finding, all subsequent studies were carried out to 8 weeks after Cre induction.

Podocyte-specific CTCF deletion causes glomerular injury and kidney failure in the absence of interstitial fibrosis. Histological analysis revealed grossly normal-appearing glomeruli at 2 weeks after Cre induction, and prominent protein casts visible throughout the renal cortex (Figure 2A). By 4 weeks, podocytes had formed adhesions to Bowman's capsule, and protein casts were prominently present throughout the cortex and the medulla (Figure 2B). Eosinophilic segmental sclerotic lesions appeared by 6 weeks after Cre induction (Figure $2 \mathrm{C}$ ), and by 8 weeks there was severe global sclerosis affecting the majority of glomeruli (Figure 2D). Dilated tubules were also noted at 8 weeks, involving primarily the renal cortex, as well as highly prominent protein casts throughout the kidney (Figure 2D). Light microscopic analysis of toluidine blue-stained semithin sections of kidney followed by electron microscopy confirmed that podocytes had undergone severe vacuolization by 4 weeks after Cre induction (Figure 3A and Supplemental Figure 3A). A detailed electron microscopic analysis showed podocyte lysis by 6 weeks after CTCF deletion (Figure 3B), which is consistent with podocyte necrosis and detachment from the glomerular basement membrane.

Turning our attention to the tubular compartment, and given the abundance of protein casts noted throughout the kidney of iCTCF ${ }^{\text {pod-/- }}$ mice (Figure 2), we stained kidney sections with an antibody directed against albumin. This revealed prominent labeling of tubular epithelial cells (Supplemental Figure 3B), suggesting avid albumin uptake, but no tubular epithelial cell loss was noted. Epithelial cells were preserved in proximal tubules with readily detectable, intact brush borders, suggesting lack of significant tubular epithelial cell injury and loss (Supplemental Figure 3B, insets). Megalin staining showed intact proximal tubular brush borders in iCTCF ${ }^{\text {pod-/- }}$ mice (Figure 3C and Supplemental Figure 4). The integrity of tubular cells was further confirmed by intact aquaporin 1 staining in kidney sections from mice at 1-8 weeks after Cre induction (Supplemental Figure 5). The investigation of the entire tubular compartment, including distal tubular and collecting duct epithelial cells, revealed comparable

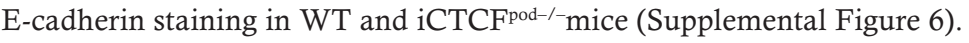

Given (a) evidence of severe glomerular pathology (Figures 2 and 3), (b) serologic evidence of advanced CKD with elevated BUN/creatinine (Figure 1), and (c) high mortality by 8-10 weeks (Figure 1), we reasoned that this animal model should allow us to address open questions about the role of interstitial fibrosis in CKD progression. In analyses performed by an experienced renal pathologist in a blinded fashion, we found no histological evidence of significant kidney fibrosis, as indicated by trichrome staining, in more than 20 mice studied (Figure 4A and Supplemental Figure 7, A and B). To confirm this, we also stained kidney sections with Jones methenamine silver (JMS), which labels collagen-rich areas of fibrosis with a prominent black stain. JMS staining and quantification did not reveal significant fibrosis even at the most advanced disease stages just prior to death (Figure 4, B and C, and Supplemental Figure 8). Consistent with the lack of fibrosis, $\alpha$-smooth muscle actin ( $\alpha$-SMA) staining was absent in interstitial cells in WT and $\mathrm{iCTCF}^{\mathrm{pod}-/-}$ mice (Figure $4 \mathrm{D}$ and Supplemental Figure 9), in contrast to robust $\alpha$-SMA staining in the positive control, a section from an 8 -week-old $C d 2 a p^{-/-}$mouse, which has significant interstitial fibrosis (Figure 4E) (19). 
A

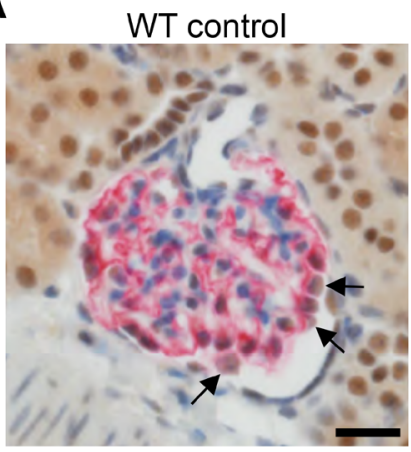

iCTCFpod--

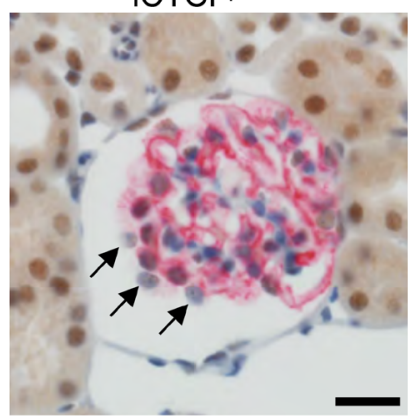

C

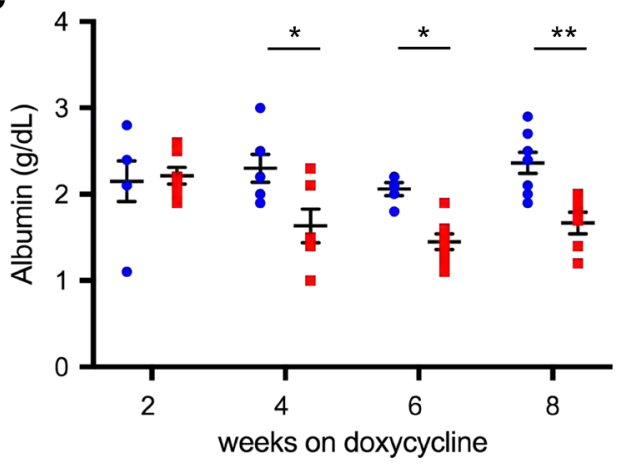

E

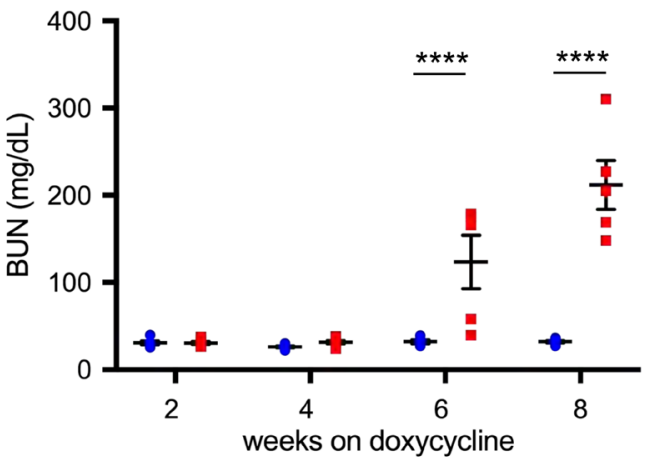

G

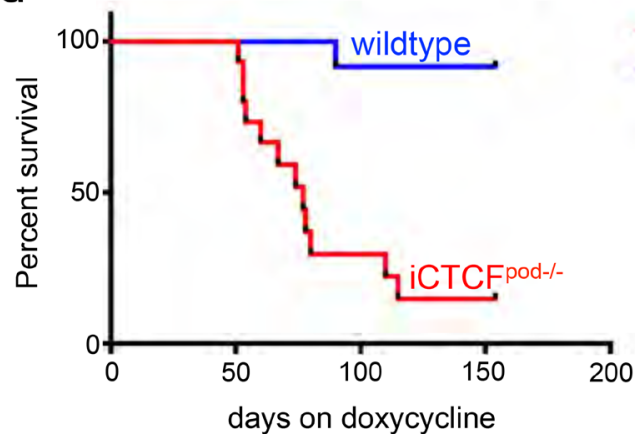

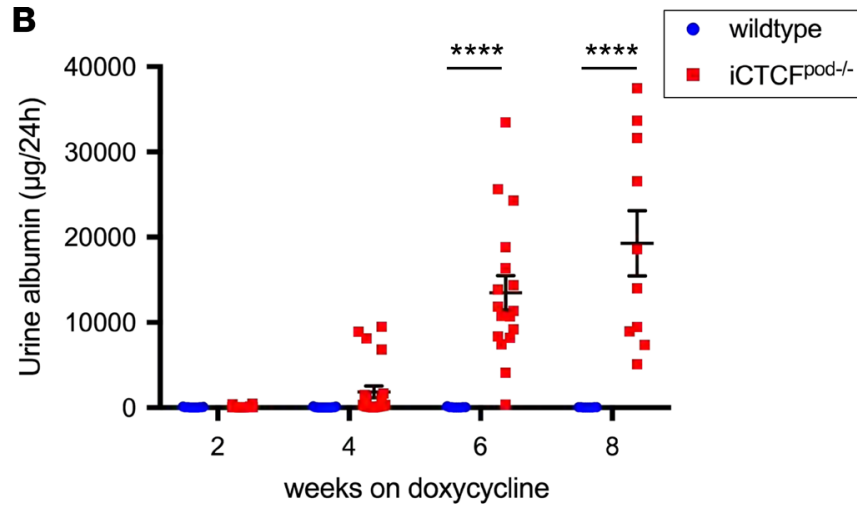

D

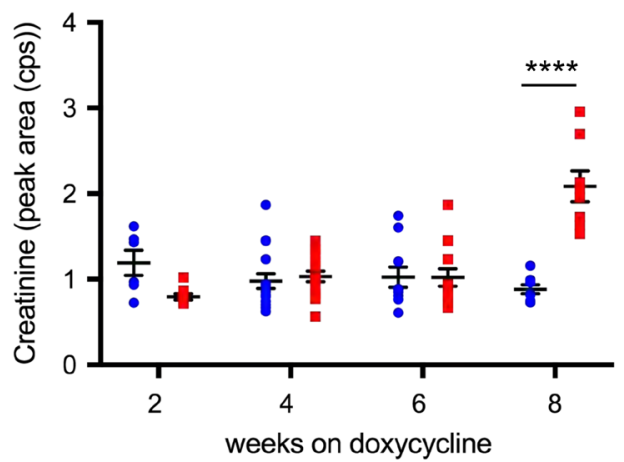

$\mathbf{F}$

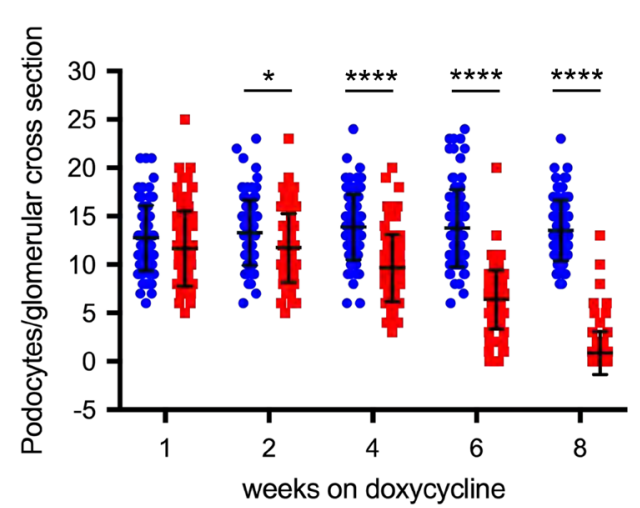

Figure 1. Podocyte-specific CTCF deletion in adult mice leads to nephrotic syndrome, podocyte loss, progressive kidney disease, and death. (A) Targeted Ctcf deletion in podocytes 1 week after Cre induction. In WT controls, arrows point to podocytes (positive for synaptopodin staining in red) replete with nuclear CTCF in brown. In iCTCFod-/- mice, arrows point to podocytes (red) devoid of brown nuclei, confirming successful deletion of CTCF specifically from podocytes. Scale bars: $20 \mu \mathrm{m}$. (B) Escalating albuminuria ( $\mu \mathrm{g} / 24$ hours) in iCTCFod-/- mice starting at 4 weeks after Cre induction. $n=13,14,21,22,9,17,9$, and 10 mice per group (left to right). (C) Serum albumin (g/dl) decreases at 4 weeks after Cre induction. For the samples shown left to right, $n=6,7,6,6,5,8,8$, and 6 mice per group. (D) Plasma creatinine is elevated by 8 weeks after Cre induction. $n=6,8,16,16,10,13,8$, and 8 mice per group (left to right). (E) BUN (mg/dl) progressively increases starting at 4 weeks after Cre induction. $n=6,7,7,7,6,5,8$, and 5 per group (left to right). (F) Podocyte quantification. For each time point, 30 glomerular cross sections from each of 3 mice were analyzed. (G) Survival curves show rapid death in iCTCFod-/- mice within 8-10 weeks after Cre induction. For WT, $n=$ 15 ; for iCTCF ${ }^{\text {pod- }- \text { - }}, n=12$. Adjusted $P$ values, controlling for multiple comparisons were calculated with a 1 -way $A N O V A$ and are reported as ${ }^{*} P<0.05$, ${ }^{* *} P<0.01$, ${ }^{* * *} P<0.0001$, comparing WT littermate controls with iCTCF ${ }^{\text {pod-/- }}$ mice at each time point. Data represent the mean \pm SEM. BUN, blood urea nitrogen. 
A
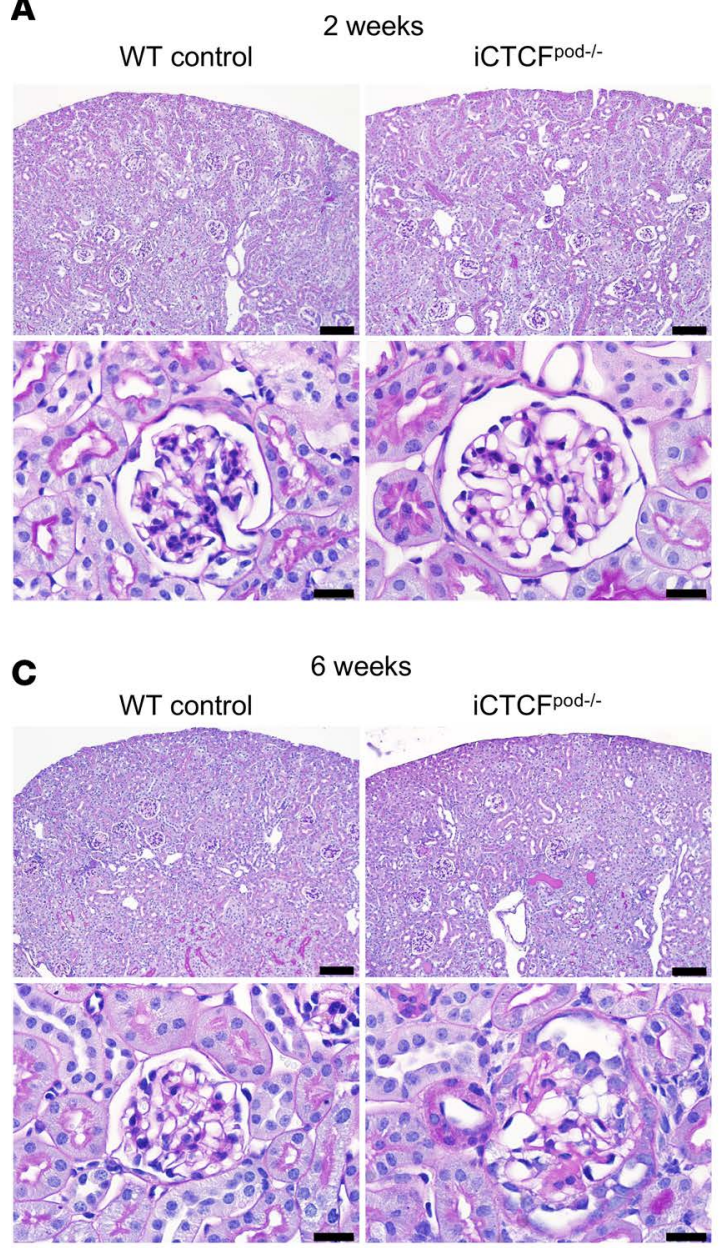

B

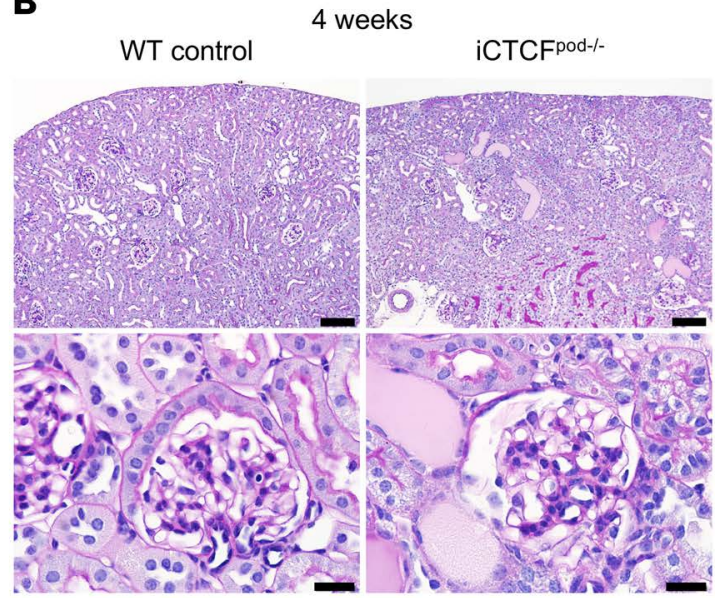

D

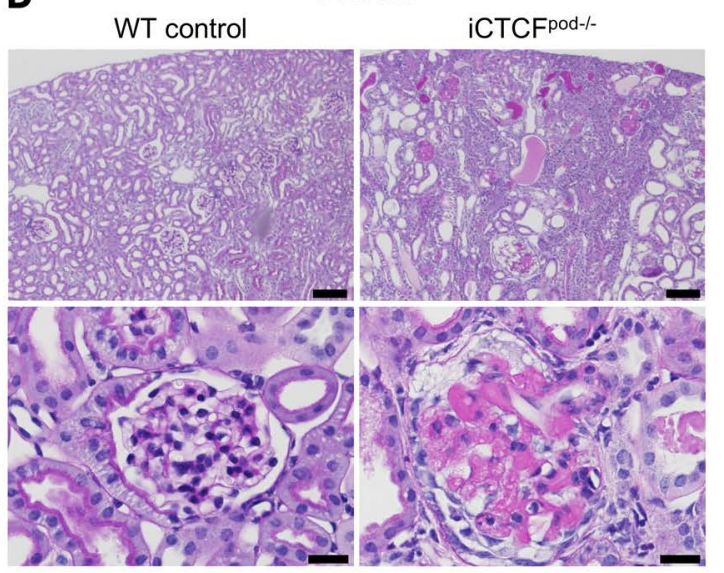

Figure 2. Progressive histopathologic changes consistent with focal and global sclerosis after podocyte ablation. Periodic acid-Schiff staining of kidney sections from control and iCTCF pod-l- mice at 2, 4, 6, and 8 weeks after Cre induction. (A) Grossly normal-appearing glomeruli at 2 weeks with prominent protein casts visible throughout the renal cortex. (B) By 4 weeks, podocytes form adhesions to Bowman's capsule, and protein casts are prominently present throughout the cortex and the medulla. (C) Eosinophilic segmental sclerotic lesions by 6 weeks. (D) By 8 weeks, severe global sclerosis affecting the majority of glomeruli, with dilated tubules involving primarily the renal cortex, as well as highly prominent protein casts throughout the kidney. Scale bars: $100 \mu \mathrm{m}$ (upper panels) and $20 \mu \mathrm{m}$ (higher-magnification lower panels).

Podocyte loss in iCTCF $F^{\text {pod-l- }}$ mice leads to FGF23 elevation and secondary HPT. Given that the timing of disease onset in $\mathrm{iCTCF}^{\mathrm{pod}-/-}$ mice is precisely defined, we next asked whether we can detect CKD-MBD in these mice, thereby allowing for close monitoring of CKD progression. We measured mineral metabolism parameters in iCTCF $^{\text {pod-/- }}$ and control mice at 2, 4, 6, and 8 weeks after Cre induction (Figure 5). In comparison with control mice, circulating FGF23 (cFGF23) levels increased progressively between 6 and 8 weeks (Figure 5A). PTH levels were significantly increased at 8 weeks after Cre induction (Figure 5B). Consistent with these findings, FGF23 gene expression in calvaria from mice at 8 weeks after Cre induction was significantly increased (Figure 5C). Compared with control mice, iCTCF ${ }^{\text {pod-l- }}$ mice also showed elevated serum phosphate levels starting at 6 weeks (Figure 5D). While total calcium levels were elevated (Figure 5E), ionized calcium (iCa) levels were significantly reduced in the iCTCF ${ }^{\text {pod-/- }}$ mice (Figure $5 \mathrm{~F}$ ), consistent with the development of secondary HPT. Total urinary excretion of phosphorus was measured, revealing a drop in urinary phosphate at 2 weeks after Cre induction, no difference at 4 weeks, and an increase at 6 weeks (Figure 5G). Consistent with increased circulating FGF23 concentrations, 1,25D levels were lower in iCTCF ${ }^{\text {pod-/- }}$ mice than in controls at 8 weeks after Cre induction (Figure $5 \mathrm{H}$ ). These changes match the onset and progression of CKD-MBD within 2-4 weeks after proteinuria, hypoalbuminemia, and podocyte loss (Figures 1 and 5).

Despite considerable FGF23 elevations, iCTCF ${ }^{\text {pd-/- }}$ animals did not develop cardiac hypertrophy or other gross cardiac abnormalities, as noted by histological analysis conducted by an expert cardiac pathologist in a 
A
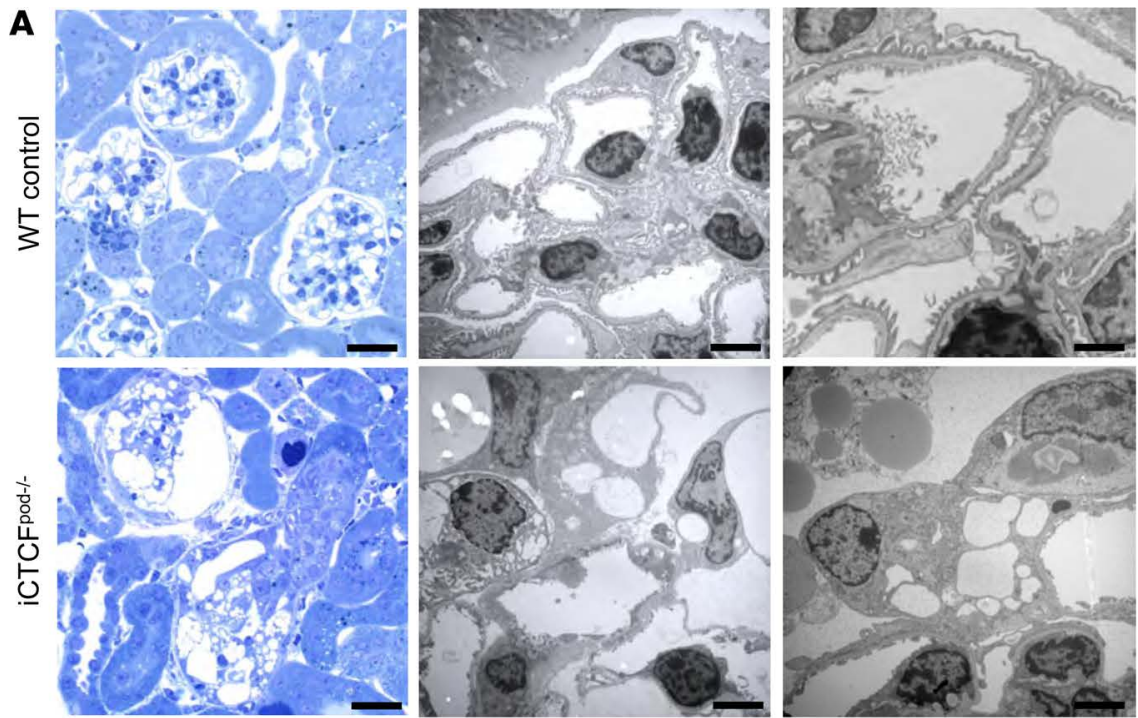

B
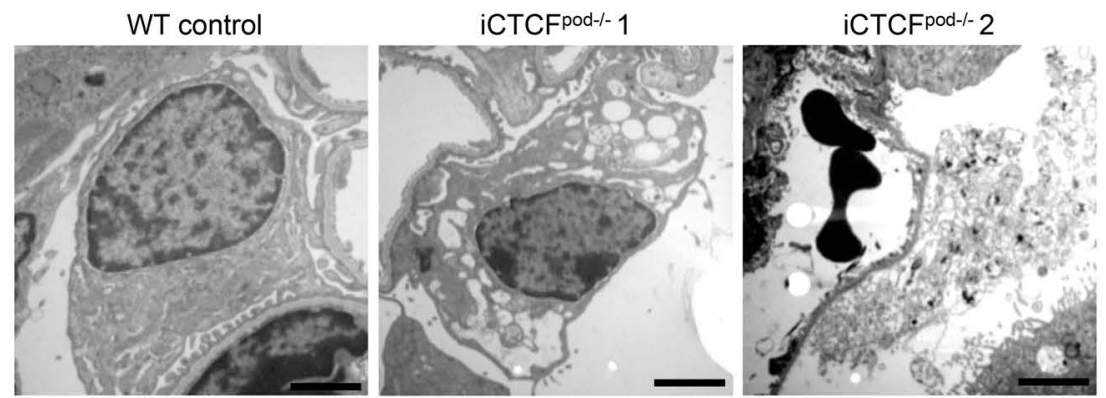

C
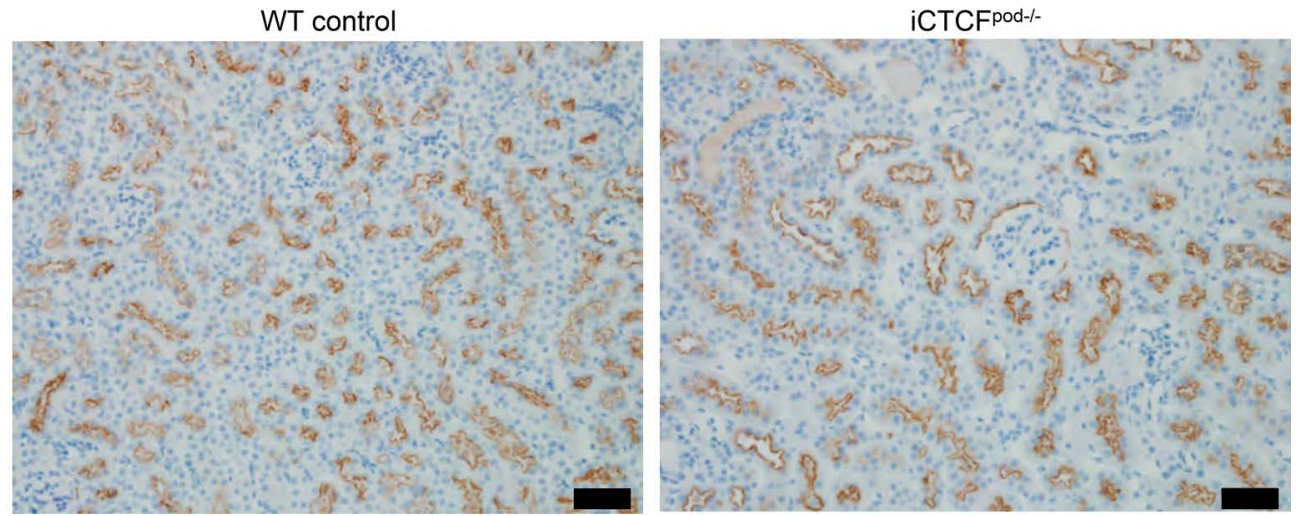

Figure 3. Inducible CTCF deletion leads to podocyte loss, characterized by vacuolization and cell lysis. (A) Toluidine blue staining (left) and corresponding transmission electron micrographs (middle, right) of kidney sections at 4 weeks after Cre induction show severe podocyte vacuolization and attenuation. Scale bars: $20 \mu \mathrm{m}$ (left), $5 \mu \mathrm{m}$ (middle), and $3 \mu \mathrm{m}$ (right). (B) Transmission electron microscopic analysis of kidney sections at 6 weeks after Cre induction identifies podocytes undergoing cell lysis as seen on the far right. Scale bars: $1 \mu \mathrm{m}$. (C) Megalin staining at 6 weeks after Cre induction shows intact proximal tubular epithelium. Scale bars: $50 \mu \mathrm{m}$.

blinded fashion (Supplemental Figure 10A). To ensure that there was no effect on the parathyroid glands, we examined parathyroid tissue from iCTCF ${ }^{\mathrm{pod}-/-}$ and control mice 8 weeks after Cre induction. Histological examination did not reveal evidence of parathyroid hyperplasia or other changes in parathyroids from iCTCF ${ }^{\text {pod-/- }}$ mice (Supplemental Figure 10B). We next sought to identify changes in gene expression profiles at 6 weeks after Cre induction for proteins involved in renal calcium and phosphate handling. We selected this time point because it marked the earliest detectable increase in BUN/creatinine. We detected decreased mRNA expression of the sodium-phosphate cotransporters NaPi2a and NaPi2c in kidneys of iCTCF ${ }^{\text {pod-l- }}$ mice (Supplemental Figure 10C). Kidney $1 \alpha$-hydroxylase (1 $\alpha \mathrm{OHase})$ and 24-hydroxylase (24OHase) expression trended higher in iCTCF ${ }^{\text {pod-/- }}$ mice and klotho mRNA expression was significantly reduced (Supplemental Figure 10C).

Podocyte loss leads to bone loss through increased resorption and osteomalacia. To evaluate the bone phenotype of iCTCF ${ }^{\text {pod-/- }}$ mice, micro-computed tomography $(\mu \mathrm{CT})$ and histomorphometry analyses were performed 
A

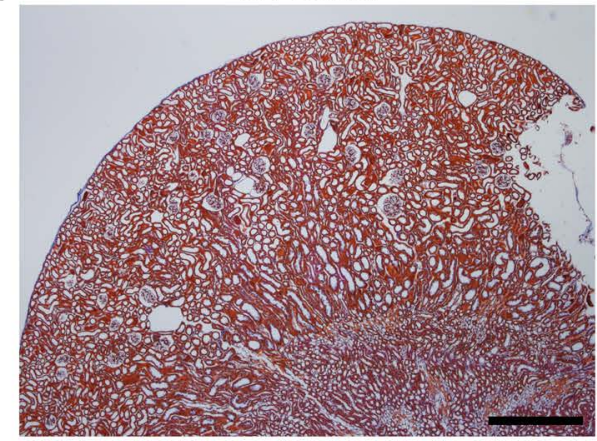

B

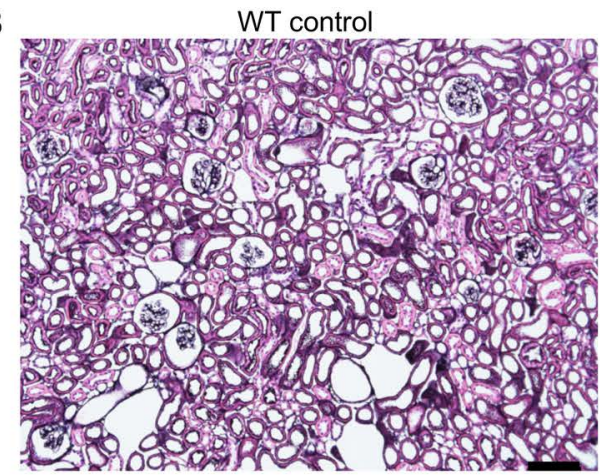

C

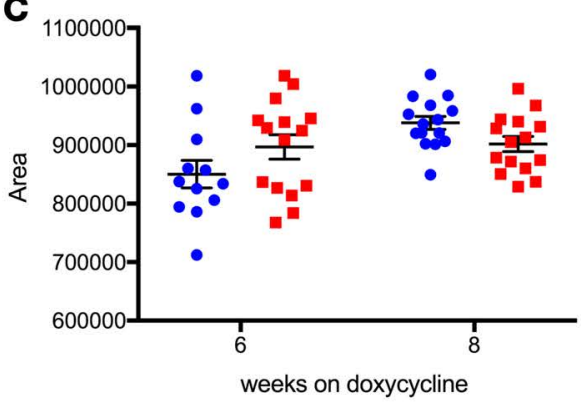

E

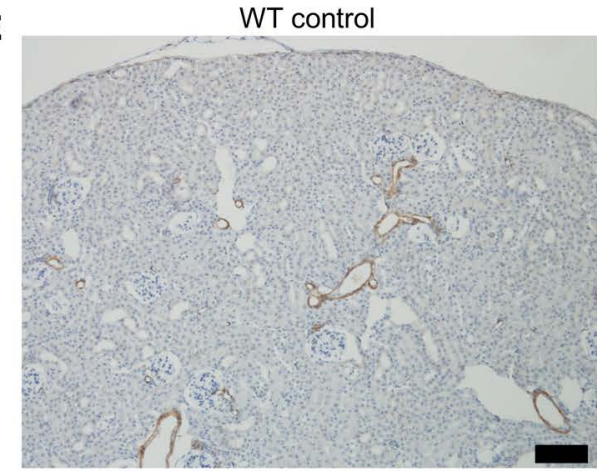

iCTCF ${ }^{\text {pod-l- }}$

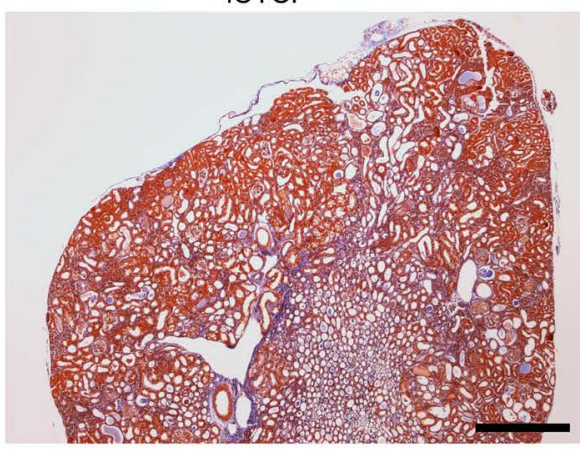

iCTCF pod- - -

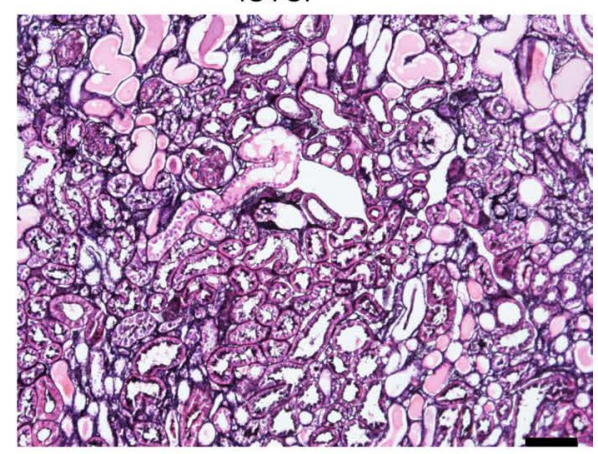

D

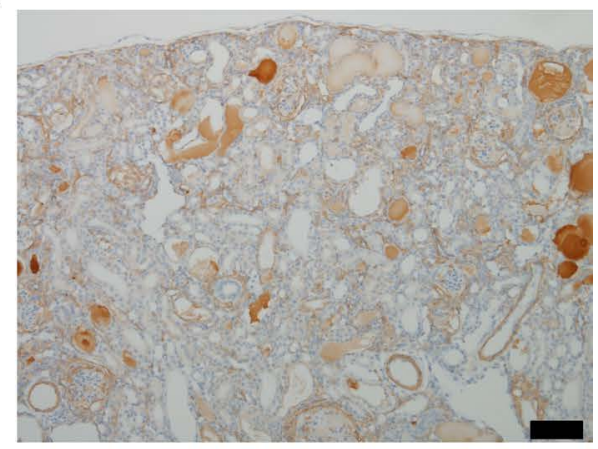

iCTCF ${ }^{\text {pod-l- }}$

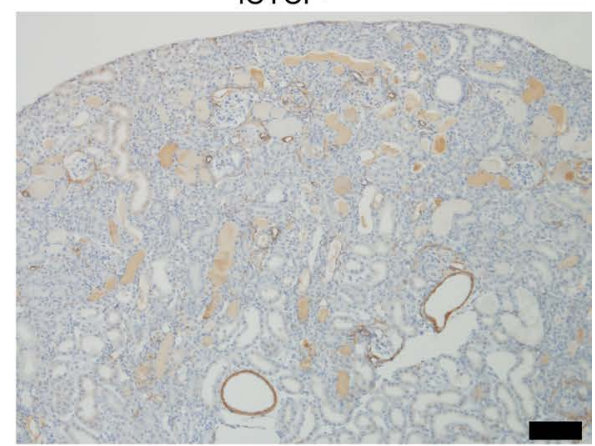

Figure 4. Podocyte ablation drives CKD in the absence of fibrosis. (A) Trichrome staining of representative sections from WT controls and iCTCF ${ }^{\text {pod }-/-}$ mice at 8 weeks after Cre induction show little to no detectable interstitial fibrosis. Scale bars: $200 \mu \mathrm{m}$. (B) Jones methenamine silver (JMS) stain (which stains collagen, reticulin, and basement membranes black) shows only focal and subtle collagen deposition in the interstitium as well as mildly thickened tubular basement membranes in iCTCF pod-/- mice that are indistinguishable from age-matched controls. Scale bars: $100 \mu \mathrm{m}$. (C) Quantification of JMS staining at 6 and 8 weeks after Cre induction reveals no significant difference between WT and iCTCF pod-/- mice. (D) Detection of interstitial $\alpha$-smooth muscle actin ( $\alpha$-SMA) staining in the kidney of an 8-week-old Cd2apknockout mouse with marked fibrosis serves as a positive control. Scale bar: $100 \mu \mathrm{m}$. (E) $\alpha$-SMA staining of kidney sections at 8 weeks after Cre induction shows similar staining patterns

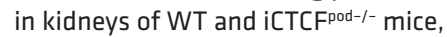
with the exception of protein casts in the iCTCF ${ }^{\text {pod-l- }}$ section, which stain brown in a nonspecific manner. Scale bars: $100 \mu \mathrm{m}$.

at 8 weeks after Cre induction. $\mu \mathrm{CT}$ analysis showed both trabecular and cortical bone loss (Table 1 and Figure 6A). Specifically, trabecular bone volume fraction (Tb.BV/TV), trabecular thickness, cortical thickness, cortical area, and cortical bone area fraction were all decreased in the $\mathrm{iCTCF}^{\mathrm{pod}-/}$ mice, while trabecular spacing was increased. This bone loss detected by $\mu \mathrm{CT}$ suggested increased susceptibility to torsional stress $\left(\mathrm{I}_{\max }\right.$ was decreased) and compromised bone strength.

Histomorphometric results were consistent in trend with the $\mu \mathrm{CT}$ data, although they did not reach significance (Table 2), including evidence for decreased BV/TV, trabecular thickness, cortical thickness, and 

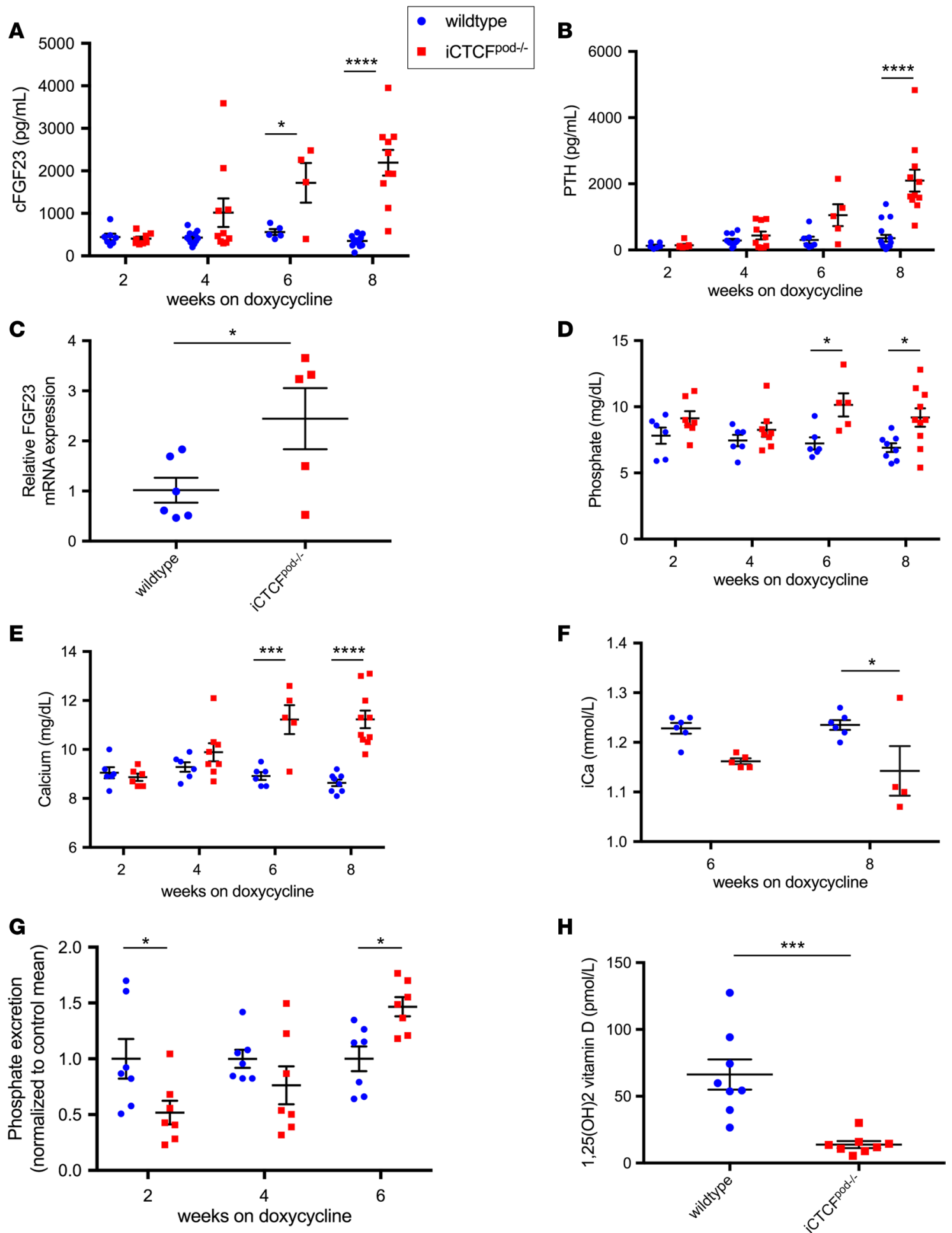

Figure 5. iCTCF ${ }^{\text {pod-/- }}$ mice have elevated FGF23, PTH, and an abnormal regulation of calcium and phosphate homeostasis. Time course of mineral metabolism measurements in iCTCF pod-/- and WT littermate control mice. Measurements were obtained 2-8 weeks after Cre induction. (A) $\mathrm{CFCF} 23$ (pg/ml). $n=7,8,11,10,5$, 4, 12, and 10 mice per group (left to right). (B) PTH (pg/ml). $n=6,7,12,10,7,5$, 16, and 11 mice per group (left to right). (C) FGF23 mRNA expression in calvaria from mice 8 weeks after Cre induction in increased more than 2 -fold in iCTCF ${ }^{\text {pod }-~}-$ mice. $n=6$ WT and 5 iCTCF ${ }^{\text {pod }-/-}$ mice. ${ }^{*} P<0.05$, unpaired $t$ test. (D) Phosphate (mg/dl). $n=6,7,6,8,6,5,8$, and 10 mice per group (left to right). (E) Calcium (mg/dl). $n=6,6,6,8,6,5,8$, and 10 mice per group (left to right). (F) iCa (mmol/l) was measured at 6 and 8 weeks after Cre induction. $n=6,5,6$, and 4 mice per group (left to right). (C) Urinary phosphate (mg/24 hours) at 2, 4, and 6 weeks after Cre induction. Values were normalized to the mean phosphate excretion for WT mice at each time point. $n=7$ mice for all groups. (H) $1,25(\mathrm{OH}) 2$ vitamin $\mathrm{D}$ (pmol/l) was measured 8 weeks after Cre induction. $n=8$ for both groups. ${ }^{* *} P<0.001$, unpaired $t$ test. Adjusted $P$ values, controlling for multiple comparisons were calculated with a 2-way ANOVA (unless otherwise noted) and are reported as ${ }^{*} P<0.05$, ${ }^{* *} P<0.001$, ${ }^{* * * *} P<0.0001$, comparing WT control with iCTCF $^{\text {pod-l- }}$ mice at each time point. Data represent the mean \pm SEM. CFGF23, circulating FGF23; PTH, parathyroid hormone; iCa, ionized calcium. 
A

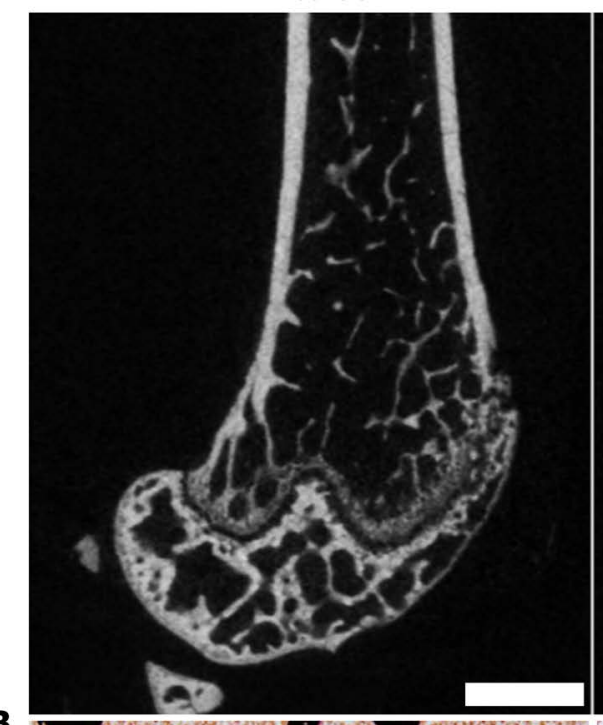

B

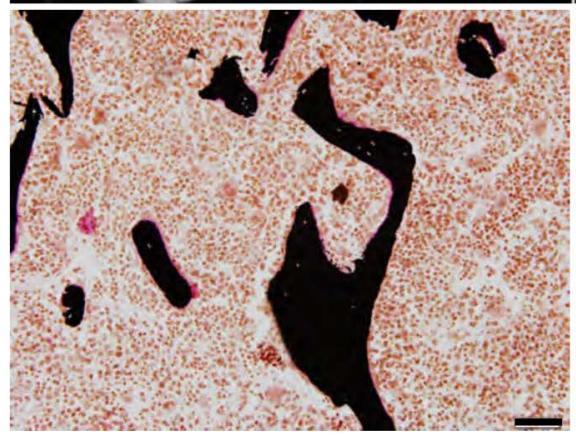

iCTCFpod- - -
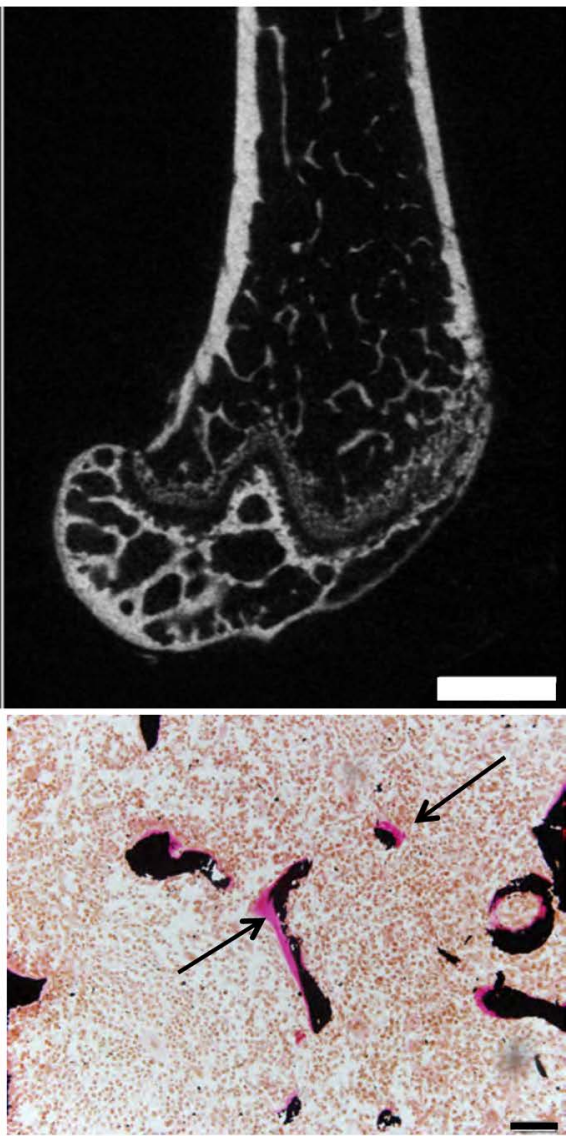

Figure 6. Bone histomorphometry reveals bone loss, osteomalacia, and increased resorption of iCTCF ${ }^{\text {pod-l- }}$ mice. (A) Sagittal images generated by $\mu \mathrm{CT}$ imaging of the distal femur of WT (left) and iCTCF ${ }^{\text {pod-/- }}$ (right) mice that were sacrificed 8 weeks after Cre induction reveal bone loss. Scale bars: $1 \mathrm{~mm}$. (B) von Kossa staining of tibias of the same WT and iCTCF ${ }^{\text {pod- }- \text { - mice reveal osteomalacia and }}$ bone loss. Arrows show unmineralized matrix. Scale bars: $20 \mu \mathrm{m}$.

increased trabecular spacing. Histomorphometry revealed a severe mineralization defect (Figure 6B), with increased osteoid/bone volume (OV/BV) and osteoid/bone surface (OS/BS) (Table 2). Cellular parameters showed increased resorption with higher eroded surface/bone surface (ES/BS), higher osteoclast surface/bone surface (OcS/BS), and higher numbers of osteoclasts/bone perimeter (N.Oc/B.Pm) (Table 2), while osteoblast parameters were not significantly different. Diffuse doxycycline labeling of bones limited dynamic measurements. Despite this limitation, we identified a trend towards reduced osteoblast activity and bone formation rates in iCTCF ${ }^{\text {pod-l- }}$ mice (Table 2).

\section{Discussion}

In this study, we show that targeted and inducible podocyte loss resulting from the podocyte-specific deletion of CTCF in adult mice causes severe nephrotic syndrome, progressive decline in renal function associated with CKD-MBD and bone loss, and premature death. The detailed characterization of this newly developed CKD model allowed us to make a number of important observations.

First, induction of podocyte loss is sufficient for the development of nephrotic syndrome and progressive CKD. This extends previous work showing that podocyte depletion through ectopic diphtheria toxin expression drives focal and segmental glomerulosclerosis (FSGS) progression in a dose-dependent manner (18). Our findings corroborate the growing understanding that podocytes are the target of choice for therapeutic interventions $(9,20-24)$, and that podocyte-preserving strategies may hold the greatest promise for CKD prevention or treatment.

Second, our model offers a potentially novel approach in which the timing of events leading to biochemical abnormalities observed in human CKD-MBD, including hyperphosphatemia and hypocalcemia, and thus elevated FGF23 and PTH levels, is readily controlled $(25,26)$. iCTCF ${ }^{\text {pod }-/-}$ mice developed a significant increase in total serum calcium levels, while, as expected, ionized calcium levels declined most likely due to the marked hyperlipidemia, consistent with findings in mice and humans $(27,28)$. The decrease in urinary phosphate excretion at 2 weeks after Cre induction is also likely related to escalating albuminuria, 
Table 1. Micro-computed tomography ( $\mu \mathrm{CT})$

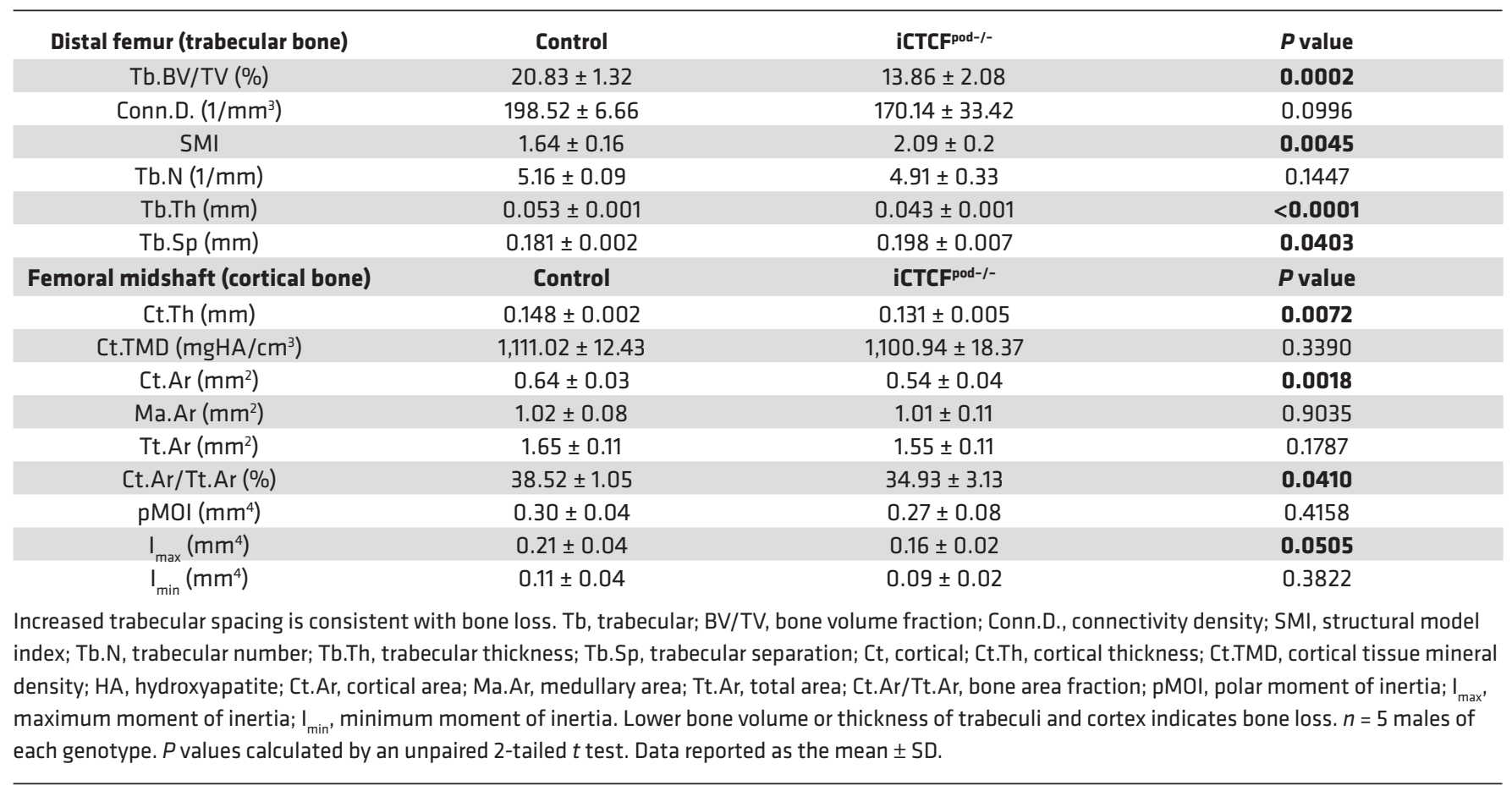

which forces proximal tubular cells to reabsorb phosphate (as well as albumin), as previously published in the puromycin aminoglycoside nephrosis model and in patients with nephrotic syndrome (29). With progressive kidney disease, $\mathrm{NaPi} 2 \mathrm{a}$ and $\mathrm{NaPi2}$ gene expression was significantly reduced in iCTCF ${ }^{\text {pod-/- }}$ mice, leading to increased urinary phosphate excretion, likely due to PTH- and FGF23-dependent mechanisms. Consistent with the conclusion that targeted podocyte loss does not impair proximal tubular cell function despite advanced $\mathrm{CKD}, 1 \alpha \mathrm{OH}$ ase gene expression trended upward, in line with previous work (30). However, the increase in $1 \alpha \mathrm{OHase}$ expression, despite a major increase in PTH levels, was insufficient to normalize 1,25D levels in iCTCF pod-l- mice, thus contributing to hypocalcemia. Elevated FGF23 levels may have effectively antagonized the PTH effect on $1 \alpha$ OHase expression. Furthermore, FGF23 may have enhanced $1,25 \mathrm{D}$ metabolism by increasing $24 \mathrm{OHase}$ expression, and the profound proteinuria in iCTCF ${ }^{\text {pod- } /-}$ mice may have led to considerable urinary losses of $1,25 \mathrm{D}$. Gene expression of klotho was dramatically reduced, which is consistent with findings in humans with advanced $\operatorname{CKD}(31,32)$. This reduction of klotho expression occurred in the absence of major histological evidence for tubular injury, suggesting that hormonal and/or other factors are involved in the regulation of klotho expression (33). Overall, our findings indicate that the action of PTH and FGF23 in the proximal tubules is preserved, revealing an additional advantage of iCTCF ${ }^{\text {pod-l- }}$ mice over toxin-induced CKD models.

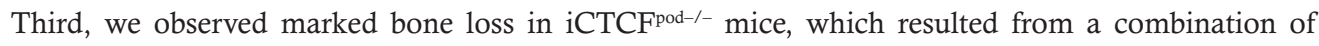
increased bone resorption and impaired mineralization of newly formed bone. While increased bone resorption can be a consequence of elevated $\mathrm{PTH}$, the presence of severe osteomalacia, as indicated by increased $\mathrm{OV} / \mathrm{BV}$, could be due to urinary loss of vitamin $\mathrm{D}$-binding protein, and thus vitamin $\mathrm{D}$, consistent with lower $1,25 \mathrm{D}$ levels in iCTCF ${ }^{\text {pod-l- }}$ mice. Previous studies reported either no change or an increase in bone volume and no defect in mineralization $(26,29)$. The absence of osteomalacia in these studies may have resulted from profoundly elevated serum phosphate levels (34), compared with modestly elevated serum phosphate levels in iCTCF ${ }^{\text {pod-I- }}$ mice. Differences in bone mineralization may also be related to the local role of FGF23 in bone, which is not well understood $(35,36)$. Osteomalacia has been reported in patients with nephrotic syndrome (37-41); the iCTCF ${ }^{\text {pod-/- }}$ mouse may therefore represent the first model in which the bone aspects of kidney disease in humans can be consistently recapitulated.

Finally, we showed that kidney fibrosis may not be required for CKD development or progression. These results dovetail with findings in Alport mice, in which the inhibition of fibrosis did not prevent CKD 
Table 2. Histomorphometry analysis

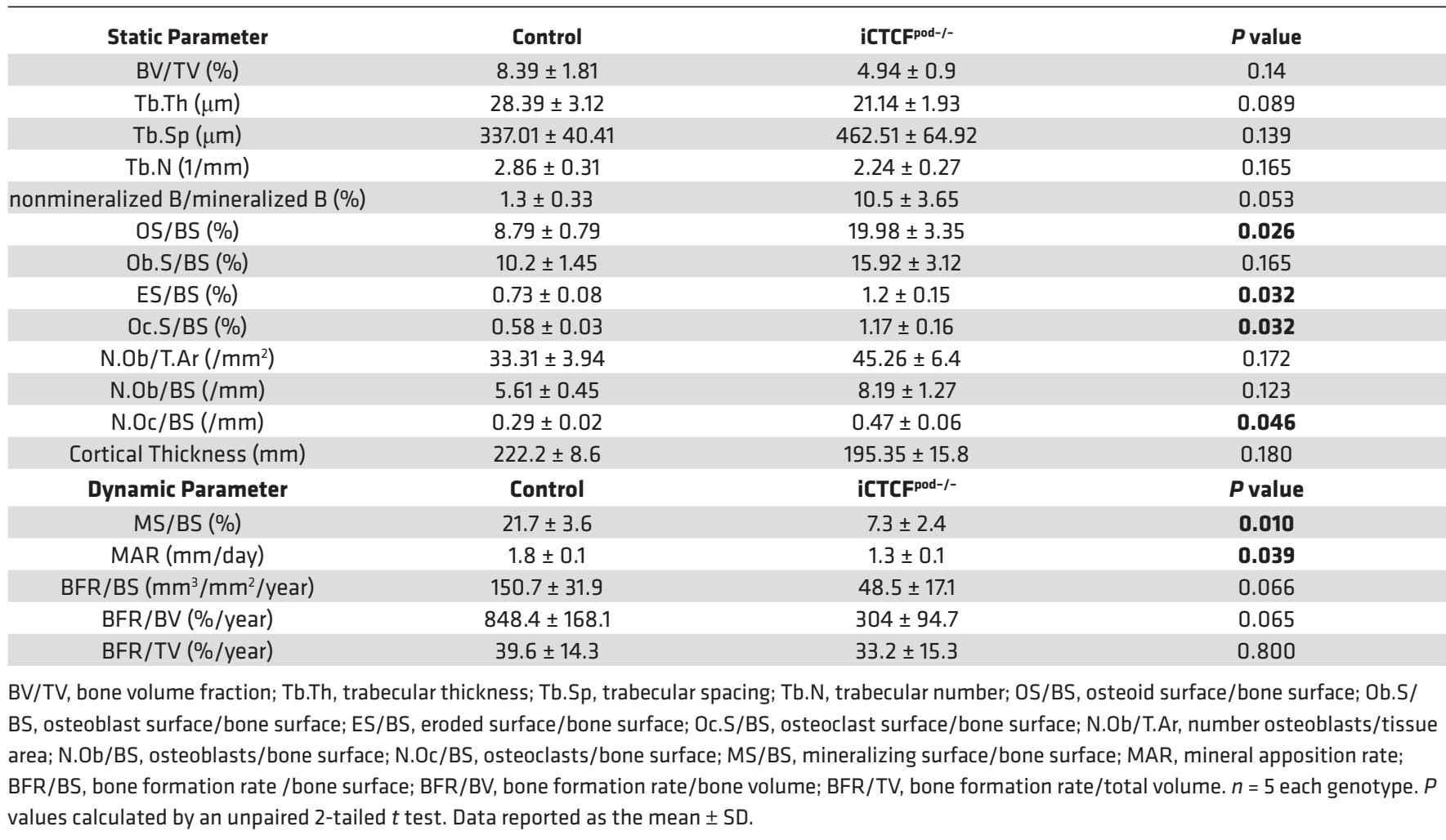

progression, because it did not fix the underlying primary glomerular defect $(42,43)$. Perhaps fibrosis is an attempt to replace a nephron damaged by primary glomerular damage (42). At advanced stages of CKD, fibrosis in patient biopsies correlates better than glomerular scarring with a decline in kidney function ( 7 , $44,45)$. However, measurements of such interstitial fibrosis may be confounded by remnants of damaged nephrons due to primary glomerular damage, such that, with the complete disappearance of nephron structures, the interstitial score artificially increases while the glomerular damage score decreases (10). While future studies will be needed to bring greater clarity to these questions, our results provide direct evidence that primary glomerular damage is sufficient to cause kidney failure in the absence of interstitial fibrosis. Our work provides experimental support for the recently proposed concept that CKD is not a singular disease entity characterized by a unifying, common mechanism, namely fibrosis, and that additional molecular diagnostic criteria are required to bring nephrology into the era of precision medicine (46).

In summary, we established and functionally characterized a mouse model of CKD generated by the inducible loss of podocytes in adult mice, which leads to nephrotic syndrome, progressive kidney failure, severe changes in mineral ion homeostasis with bone disease, and ultimately death from renal failure in the absence of interstitial kidney fibrosis. Our work may provide the foundation for future studies to better understand kidney disease progression and to explore the efficacy of much needed therapeutic approaches targeting the podocyte population.

\section{Methods}

Animal care. Both male and female on a mixed C57BL6/129 background were used. No differences in measurements were revealed between the sexes in pilot experiments. Sample sizes were at least 4 and generally 5-8 and as high as 22 mice per group. Doxycycline (4 g/1) (Sigma-Aldrich, D9891) was continuously adminis-

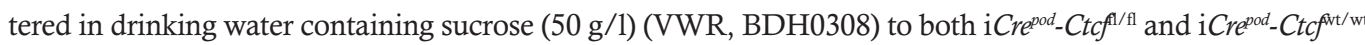
littermate control mice (age 6 weeks at start of doxycycline) to drive Cre expression specifically in podocytes. Mice were sacrificed at prespecified time points, after start of doxycycline by isoflurane inhalation and cervical dislocation. Prior to sacrifice, blood was obtained by cardiac puncture or tail bleeding. Tissues were 
harvested and snap frozen in liquid nitrogen for mRNA isolation or fixed in formalin or $70 \%$ ethanol depending on the analysis and stored at $4^{\circ} \mathrm{C}$. A subset of mice was placed in metabolic cages for urine collection for 24 hours. Urine analysis for albuminuria was performed as previously described (47).

Biochemical and hormonal measurements. Plasma levels of albumin, calcium, phosphorus, and urea nitrogen were measured using an automated bioanalyzer (Heska DriChem 7000). Serum creatinine was measured by liquid chromatography, mass spectrometry (48). Plasma levels of PTH, C-terminal FGF23 (Immutopics) and 1,25D (IDS) were measured by ELISA according to the manufacturers' instructions. Urine calcium and phosphorus were measured using commercial kits from Stanbio.

Histological analysis. Kidney histology of at least 5 mice from each group was assessed. Light microscopy images of periodic acid-Schiff (PAS), trichrome, JMS, or toluidine blue-stained sections as well as transmission electron micrographs were analyzed in a blinded fashion by A. Weins and classified using standard criteria. JMS staining was quantified using ImageJ (version 1.50i, NIH) by converting color images to 8-bit and then binary and measuring black areas. Immunoperoxidase double staining for CTCF and synaptopodin was performed according to standard protocols using CTCF (Cell Signaling Technology, 3418) and synaptopodin (49) antibodies. Podocyte quantification was performed on formalin-fixed, paraffin-embedded tissue sections using transducin-like enhancer of split 4 (TLE4) (50). Immunoperoxidase staining for albumin (DakoCytomation, F0117), LRP2/megalin (Abcam, ab76969), aquaporin 1 (Abcam, ab15080), E-cadherin (Abcam, ab 76055), or $\alpha$-SMA (Abcam, ab7817) was performed according to standard protocols. Sections of formalinfixed, paraffin-embedded parathyroid tissue were stained with hematoxylin and eosin (H\&E) and evaluated by light microscopy. Sections of formalin-fixed, paraffin-embedded heart tissue were stained with $\mathrm{H} \& \mathrm{E}$ and evaluated by light microscopy by a cardiac pathologist (Frederick Schoen, Brigham and Women's Hospital).

Quantification of JMS staining. Color images of JMS-stained kidney sections were converted to 8-bit and then binary using ImageJ software. Areas of black staining were quantified using ImageJ and statistical significance evaluated by a 2-way ANOVA test.

Histomorphometric analysis of bone. Mice were injected intraperitoneally with $20 \mathrm{mg} / \mathrm{kg}$ calcein and 40 $\mathrm{mg} / \mathrm{kg}$ alizarin complexone on days 7 and 2 before sacrifice, respectively. Femurs were isolated, cleaned of adherent tissues, and fixed in $70 \%$ ethanol. Bones were then dehydrated, infiltrated, and embedded in methyl methacrylate. Consecutive undecalcified $4-\mu \mathrm{m}$-thick sections were stained by von Kossa with van Gieson counterstain and toluidine blue to determine structural and cellular parameters, respectively. Dynamic parameters were quantified using unstained, undecalcified sections. Histomorphometric parameters were measured using the Osteomeasure image analysis system coupled to a microscope (BX50 combined with a DP72 digital camera, Olympus). A sampling site of approximately $2 \mathrm{~mm}^{2}$ was established in the cancellous bone at $400 \mu \mathrm{m}$ below the growth plate. Analysis was done in a standardized fashion by an experienced scientist and results were expressed according to the updated standardized ASBMR nomenclature (51).

$\mu C T$. $\mu \mathrm{CT}$ imaging was performed on the distal metaphysis and mid-diaphysis of the femur of mice after 8 weeks on doxycycline using a high-resolution desktop imaging system ( $\mu$ CT40, Scanco Medical AG). Scans were acquired using a $10-\mu \mathrm{m}^{3}$ isotropic voxel size, $70-\mathrm{kVp}$ peak x-ray tube potential, 200-ms integration time, and were subjected to Gaussian filtration. Trabecular and cortical bone architecture were measured using the standard trabecular and cortical bone morphology scripts in the Scanco $\mu$ CT Evaluation Program. Trabecular bone microarchitecture was evaluated in the distal metaphysis in a region that began $200 \mu \mathrm{m}$ (20 slices) above the peak of the distal growth plate and extended proximally $1.5 \mathrm{~mm}$ (150 slices). Cortical bone was evaluated in the mid-diaphysis in a region that started $55 \%$ of the bone length below the femoral head and extended $500 \mu \mathrm{m}$ (50 slices) distally. Segmentation thresholds of 247 and 696 $\mathrm{mg}$ hydroxyapatite $/ \mathrm{cm}^{3}$ were used for the evaluations of trabecular and cortical bone, respectively, based on adaptive-iterative thresholding (AIT) that was performed on the iCTCF ${ }^{\text {pod-l- }}$ group.

Gene expression analysis. mRNA was isolated from total kidneys or from calvaria using the TRIzol method and purified using Purelink (Ambion) and reverse transcribed using a Qiagen quantiTect RT kit. Primer sequences used are listed in Supplemental Table 1.

Liquid chromatography, mass spectrometry-based lipid profiling. Lipids were profiled as previously described (52). In brief, $10 \mu 1$ of mouse serum was extracted with $190 \mu 1$ of isopropanol containing an internal standard, 1-dodecanoyl-2-tridecanoyl-sn-glycero-3-phosphocholine (Avanti Polar Lipids). After centrifugation, supernatants were injected directly, followed by reverse-phase chromatography using a $150 \times 3.0 \mathrm{~mm}$ Prosphere HP C4 column (Grace). Mobile phase A consisted of 95:5:0.1 (v/v/v) $10 \mathrm{mM}$ ammonium acetate/ methanol/acetic acid; mobile phase B consisted of 99.9:0.1 (v/v) methanol/acetic acid. The column was 
eluted isocratically with $80 \%$ mobile phase A for 2 minutes followed by a linear gradient to $20 \%$ mobile phase A over 1 minute, a linear gradient to $0 \%$ mobile phase A over 12 minutes, then 10 minutes at $0 \%$ mobile phase A. Analyses were carried out using a 4000 QTRAP triple quadrupole mass spectrometer (Applied Biosystems/Sciex) with electrospray ionization and Q1 scans in the positive ion mode. Internal standard peak areas were monitored for quality control and used to normalize analyte peak areas. For each lipid analyte, the first number denotes the total number of carbons in the lipid acyl chain(s) and the second number (after the colon) denotes the total number of double bonds in the lipid acyl chain(s).

Statistics. PRISM software (GraphPad) was used for statistical analysis, and comparisons between groups were evaluated by unpaired, 2-tailed t-test or 2-way ANOVA where applicable. $P$ values were adjusted for multiple comparisons with a Bonferroni correction. $P$ values and adjusted $P$ values of less than 0.05 were considered significant.

Study approval. All experiments were approved by the Institutional Animal Care and Use Committee at Massachusetts General Hospital and Brigham and Women's Hospital.

\section{Author contributions}

$\mathrm{MC}, \mathrm{ARC}, \mathrm{AW}, \mathrm{SH}, \mathrm{PM}, \mathrm{HJ}$, and AG designed and performed the experiments. BC, EPR, HS, DB, EH, JYJ, and MB performed experiments. NG provided advice on the generation of podocyte-specific CTCF mice. HJ, PM, and AG supervised the project. MC, ARC, PM, HJ, and AG wrote the manuscript. All authors read the manuscript and provided feedback.

\section{Acknowledgments}

The authors wish to thank Terri Woo and Colleen Ford in the Brigham and Women's Hospital Pathology Department for technical assistance. We are grateful to Frederick Schoen of Brigham and Women's Hospital Cardiac Pathology for the histological examination of cardiac sections. We wish to also thank Marcus Moeller (University of Aachen, Germany) for providing podocyte-specific Cre deleter mice. M. Christov was supported by NIH grant DK093608 and the New York Community Trust; P. Mundel by NIH grants DK057683, DK062472, and DK091218; H. Jüppner by NIH grants DK46718-24 and P01 DK11794; A. Greka by NIH grants DK099465, DK083511, DK093746, and DK095045; and A. Weins by NIH grants DK093783 and DK107918. $\mu$ CT was performed by the Center for Skeletal Research Core (NIH P30 AR066261).

Address correspondence to: Anna Greka, Harvard Institutes of Medicine, 4 Blackfan Circle, Boston, Massachusetts 02115, USA or The Broad Institute of MIT and Harvard, 415 Main Street, Cambridge, Massachusetts 02142, USA. Phone: 617.525.5939; Email: agreka@bwh.harvard.edu or agreka@broadinstitute.org. Or to: Marta Christov, New York Medical College, Basic Science Building, Room C06, Valhalla, New York 10595, USA. Phone: 914.493.7701; Email: marta_christov@nymc.edu.

PM's present address is: Goldfinch Biopharma, Cambridge, Massachusetts, USA.

1. GBD 2015 Mortality Causes of Death Collaborators. Global, regional, and national life expectancy, all-cause mortality, and cause-specific mortality for 249 causes of death, 1980-2015: a systematic analysis for the Global Burden of Disease Study 2015. Lancet. 2016;388(10053):1459-1544.

2. Levey AS, Coresh J. Chronic kidney disease. Lancet. 2012;379(9811):165-180.

3. Chawla LS, Eggers PW, Star RA, Kimmel PL. Acute kidney injury and chronic kidney disease as interconnected syndromes. N Engl J Med. 2014;371(1):58-66.

4. Isakova T, et al. Rationale and approaches to phosphate and fibroblast growth factor 23 reduction in CKD. J Am Soc Nephrol. 2015;26(10):2328-2339.

5. Kim SM, Long J, Montez-Rath M, Leonard M, Chertow GM. Hip fracture in patients with non-dialysis-requiring chronic kidney disease. J Bone Miner Res. 2016;31(10):1803-1809.

6. Denburg MR, et al. Fracture burden and risk factors in childhood CKD: results from the CKiD cohort study. J Am Soc Nephrol. 2016;27(2):543-550.

7. Duffield JS. Cellular and molecular mechanisms in kidney fibrosis. J Clin Invest. 2014;124(6):2299-2306.

8. Ferenbach DA, Bonventre JV. Kidney tubules: intertubular, vascular, and glomerular cross-talk. Curr Opin Nephrol Hypertens. 2016;25(3):194-202.

9. Greka A, Mundel P. Cell biology and pathology of podocytes. Annu Rev Physiol. 2012;74:299-323.

10. Kriz W. Progression of chronic renal failure in focal segmental glomerulosclerosis: consequence of podocyte damage or of tubulointerstitial fibrosis? Pediatr Nephrol. 2003;18(7):617-622.

11. Yang L, Besschetnova TY, Brooks CR, Shah JV, Bonventre JV. Epithelial cell cycle arrest in G2/M mediates kidney fibrosis after 
injury. Nat Med. 2010;16(5):535-543.

12. Shobeiri N, Adams MA, Holden RM. Vascular calcification in animal models of CKD: A review. Am J Nephrol. 2010;31(6):471-481.

13. Caulfield JP, Reid JJ, Farquhar MG. Alterations of the glomerular epithelium in acute aminonucleoside nephrosis. Evidence for formation of occluding junctions and epithelial cell detachment. Lab Invest. 1976;34(1):43-59.

14. Jia T, et al. A novel model of adenine-induced tubulointerstitial nephropathy in mice. BMC Nephrol. 2013;14:116.

15. Dekker J, Mirny L. The 3D genome as moderator of chromosomal communication. Cell. 2016;164(6):1110-1121.

16. Phillips JE, Corces VG. CTCF: master weaver of the genome. Cell. 2009;137(7):1194-1211.

17. Soshnikova N, Montavon T, Leleu M, Galjart N, Duboule D. Functional analysis of CTCF during mammalian limb development. Dev Cell. 2010;19(6):819-830.

18. Wharram BL, et al. Podocyte depletion causes glomerulosclerosis: diphtheria toxin-induced podocyte depletion in rats expressing human diphtheria toxin receptor transgene. J Am Soc Nephrol. 2005;16(10):2941-2952.

19. Weins A, et al. Dendrin ablation prolongs life span by delaying kidney failure. Am J Pathol. 2015;185(8):2143-2157.

20. Yu CC, et al. Abatacept in B7-1-positive proteinuric kidney disease. N Engl J Med. 2013;369(25):2416-2423.

21. Mundel P, Greka A. Developing therapeutic 'arrows' with the precision of William Tell: the time has come for targeted therapies in kidney disease. Curr Opin Nephrol Hypertens. 2015;24(4):388-392.

22. Faul C, et al. The actin cytoskeleton of kidney podocytes is a direct target of the antiproteinuric effect of cyclosporine A. Nat Med. 2008;14(9):931-938.

23. Zhou Y, et al. A small-molecule inhibitor of TRPC5 ion channels suppresses progressive kidney disease in animal models. Science. 2017;358(6368):1332-1336.

24. Sieber J, et al. GDC-0879, a BRAFV600E inhibitor, protects kidney podocytes from death [published online ahead of print December 7, 2017]. Cell Chem Biol. https://doi.org/10.1016/j.chembiol.2017.11.006.

25. Moe SM, et al. A rat model of chronic kidney disease-mineral bone disorder. Kidney Int. 2009;75(2):176-184.

26. Stubbs JR, et al. Longitudinal evaluation of FGF23 changes and mineral metabolism abnormalities in a mouse model of chronic kidney disease. J Bone Miner Res. 2012;27(1):38-46.

27. Fang Y, Ginsberg C, Sugatani T, Monier-Faugere MC, Malluche H, Hruska KA. Early chronic kidney disease-mineral bone disorder stimulates vascular calcification. Kidney Int. 2014;85(1):142-150.

28. Sachmechi I, Shah G, Rezainadimi L, Blaustein DA, Rosner F. Misleading acute hypercalcemia due to hyperlipidemia: a method-dependent error. Endocr Pract. 1997;3(5):293-296.

29. de Seigneux S, et al. Proteinuria increases plasma phosphate by altering its tubular handling. J Am Soc Nephrol. 2015;26(7):1608-1618.

30. Helvig CF, et al. Dysregulation of renal vitamin D metabolism in the uremic rat. Kidney Int. 2010;78(5):463-472.

31. Koh N, et al. Severely reduced production of klotho in human chronic renal failure kidney. Biochem Biophys Res Commun. 2001;280(4):1015-1020.

32. Asai $\mathrm{O}$, et al. Decreased renal $\alpha$-Klotho expression in early diabetic nephropathy in humans and mice and its possible role in urinary calcium excretion. Kidney Int. 2012;81(6):539-547.

33. Neyra JA, Hu MC. Potential application of klotho in human chronic kidney disease. Bone. 2017;100:41-49.

34. Sabbagh Y, et al. Repression of osteocyte Wnt/ $\beta$-catenin signaling is an early event in the progression of renal osteodystrophy J Bone Miner Res. 2012;27(8):1757-1772.

35. Shimada T, et al. Targeted ablation of Fgf23 demonstrates an essential physiological role of FGF23 in phosphate and vitamin D metabolism. J Clin Invest. 2004;113(4):561-568.

36. Wesseling-Perry K, et al. Relationship between plasma fibroblast growth factor- 23 concentration and bone mineralization in children with renal failure on peritoneal dialysis. J Clin Endocrinol Metab. 2009;94(2):511-517.

37. Mittal SK, Dash SC, Tiwari SC, Agarwal SK, Saxena S, Fishbane S. Bone histology in patients with nephrotic syndrome and normal renal function. Kidney Int. 1999;55(5):1912-1919.

38. Freundlich M, Jofe M, Goodman WG, Salusky IB. Bone histology in steroid-treated children with non-azotemic nephrotic syndrome. Pediatr Nephrol. 2004;19(4):400-407.

39. Malluche HH, Goldstein DA, Massry SG. Osteomalacia and hyperparathyroid bone disease in patients with nephrotic syndrome. J Clin Invest. 1979;63(3):494-500.

40. Malluche HH, Mawad HW, Monier-Faugere MC. Renal osteodystrophy in the first decade of the new millennium: analysis of 630 bone biopsies in black and white patients. J Bone Miner Res. 2011;26(6):1368-1376

41. Wesseling-Perry K, et al. Early skeletal and biochemical alterations in pediatric chronic kidney disease. Clin J Am Soc Nephrol. 2012;7(1):146-152.

42. Hahm K, et al. Alphav beta6 integrin regulates renal fibrosis and inflammation in Alport mouse. Am J Pathol. 2007;170(1):110-125

43. Ninichuk V, et al. Multipotent mesenchymal stem cells reduce interstitial fibrosis but do not delay progression of chronic kidney disease in collagen4A3-deficient mice. Kidney Int. 2006;70(1):121-129.

44. Bonventre JV. Can we target tubular damage to prevent renal function decline in diabetes? Semin Nephrol. 2012;32(5):452-462.

45. Zeisberg M, Neilson EG. Mechanisms of tubulointerstitial fibrosis. J Am Soc Nephrol. 2010;21(11):1819-1834.

46. Hall YN, Himmelfarb J. The CKD classification system in the precision medicine era. Clin J Am Soc Nephrol. 2017;12(2):346-348.

47. Schaldecker T, et al. Inhibition of the TRPC5 ion channel protects the kidney filter. J Clin Invest. 2013;123(12):5298-5309.

48. Rhee EP, et al. Metabolite profiling identifies markers of uremia. J Am Soc Nephrol. 2010;21(6):1041-1051.

49. Mundel P, Heid HW, Mundel TM, Krüger M, Reiser J, Kriz W. Synaptopodin: an actin-associated protein in telencephalic dendrites and renal podocytes. J Cell Biol. 1997;139(1):193-204.

50. Venkatareddy M, et al. Estimating podocyte number and density using a single histologic section. J Am Soc Nephrol. 2014;25(5):1118-1129.

51. Dempster DW, et al. Standardized nomenclature, symbols, and units for bone histomorphometry: a 2012 update of the report of the ASBMR Histomorphometry Nomenclature Committee. J Bone Miner Res. 2013;28(1):2-17.

52. Rhee EP, et al. Lipid profiling identifies a triacylglycerol signature of insulin resistance and improves diabetes prediction in humans. J Clin Invest. 2011;121(4):1402-1411. 